\title{
Resonant ultrasound spectroscopy for investigation of thin surface coatings
}

\author{
H. Seiner ${ }^{1,2}$, M. Růžek ${ }^{1,2}$, P. Sedlák ${ }^{1,2}$, L. Bicanová ${ }^{1,2}$ \& M. Landa ${ }^{1}$ \\ ${ }^{1}$ Institute of Thermomechanics ASCR, Czech Republic \\ ${ }^{2}$ Czech Technical University in Prague, Czech Republic
}

\begin{abstract}
In this paper, we analyze the possibility of determination of all in-plane independent elastic coefficients of an anisotropic surface coating on a known substrate by means of resonant ultrasound spectroscopy (RUS). A novel approach based on perturbation theory is presented, which enables direct determination of the elastic coefficients of the coating from the shift of resonant frequencies induced by the deposition of the layer. The reliability of the proposed concept is investigated by numerical simulations as well as verified by experiments.
\end{abstract}

Keywords: ultrasound spectroscopy, thin coatings, perturbation theory, non-destructive evaluation, in-plane anisotropy.

\section{Introduction}

Reliable determination of elastic coefficients of thin surface layers and coatings is one of the most challenging topics of today's experimental mechanics. Conventional micro/nanoindentation techniques are usually neither able to distinguish clearly which part of the elastic response is inherent to the coating and which to the underlying substrate, nor suitable for determination of all independent elastic coefficients of anisotropic coatings. A promising alternative to these highly local techniques are the ultrasonic methods, which may be either the methods based on surface elastic waves (SAW), or the resonant ultrasound spectroscopy (RUS) where the sought elastic coefficients are determined from measurements of resonant spectra of free elastic vibrations of a specimen of the examined material. The latter will be discussed in this paper.

Nowadays, the RUS is one of the most widely used methods for determination of elastic coefficients of bulk anisotropic materials. This method has found its very first applications in geophysics as early as in late 1960s, where the elas- 
tic coefficients of rocks and minerals were determined from resonant spectra of spherical samples (so-called resonant sphere technique, RST [1]) or small cubes [2] of the examined material. Later on, the method was significantly improved by Ohno and his coworkers, enabling determination of anisotropic elastic coefficients up to orthorhombic [3] and trigonal [4] symmetry class from measurements on a rectangular parallelepiped (so-called rectangular parallelepiped resonance, RPR). In early 1990s, Migliori et al. [5] introduced this method to solid state physics community, and applied it immediately to in-situ monitoring of elasticity changes of high temperature superconductors when undergoing transitions to the superconducting state [6]. In these pioneering works, also the more general abbreviation RUS started to be used. The measurements were further improved by scanning the surface of the resonating specimen by a laser vibrometer, thus not only the resonant frequencies but also the shapes of vibration modes became available. In 2002, Ogi et al. [7] used this improvement for reliable identification of individual modes in the obtained spectrum, which was the approach further developed by the authors in the past few years [8-12].

As far as the investigation of thin surface layers and coatings is concerned, the first attempt to modify the RUS technique for such problems can be found in Visscher's paper [13], where, among many other general shapes of the specimen, also a 'sandwich' (a specimen consisting of two parallel layers) is discussed. By decreasing the thickness of one of the layers, such 'sandwich' obviously limits to a substrate-coating system; this idea was utilized by So et al. [14], who used the concept outlined in [13] for modeling of free vibrations of a $\mathrm{SrTiO}_{3}$ substrate coated by a thin magnetoresistant film.

Extensive experimental literature on RUS investigation of thin surface layers was published by Ogi and his coworkers. It covers a huge variety of substratecoating systems ranging from superlattice thin films ( $\mathrm{Co} / \mathrm{Pt}$ multilayer in [15]) to diamond coatings deposited by CVD [16,17]. The main improvement brought by the Ogi's group to the experimental embodiment of RUS lies in a novel experimental scheme, so-called tripod scheme (e.g. [7]). In this scheme, the investigated specimen is freely laid in a tripod of thin, rod-like piezoelectric transducers (one of them is used for generation of vibrations, the others for detection of the specimen's response). Compared to the classical scheme used in earlier works (e.g. [5]), where the specimen is clamped between two transducers, the tripod scheme ensures that the vibrations of the specimen are very close to free vibrations (the clamping force is eliminated, which is crucial particularly for thin plates or shells). For mathematical description of the vibrating substrate-coating systems, the Ogi's group uses full 3D-3D 'sandwich' models based on the concept of Visscher [13], often utilizing an interpolation method developed by Heyliger [18].

In this paper, novel theoretical and the experimental approaches will be discussed. Unlike to the 'sandwich' concept from [13], our approach reflects the multiscale character of the problem, treating the surface layer as a 2D object deposed on a 3D substrate. In the experimental part, a fully non-contact setup [11] is presented, where the vibrations of the specimen are both generated and detected by lasers. As it will be shown, these novel approaches lead to reliable, reproducible 
and straightforward determination of elastic coefficients of the examined surface layer.

\section{Theory and preliminary numerical tests}

The main idea of RUS measurements is following: First, the resonant spectrum of vibrations of the examined specimen is measured and individual resonant frequencies are localized. Then, the elastic coefficients are determined inversely, which means that they are tuned such that the resonant frequencies computed for them fit the experimentally obtained spectrum in some optimal way. For such procedure, one must be able to solve the so-called direct problem, i.e. to evaluate the resonant frequencies of the specimen for known elastic coefficients. For homogeneous, bulk specimens of simple geometry, this can be easily achieved by finding stationary points of the Lagrangian energy of the vibrating specimen (a simple variational problem, which can be easily solved by Ritz method, see e.g. [5, 7, 10, 12]). In the following section, the direct problem will be posted and solved for a substratecoating system.

\subsection{Perturbation model of a vibrating substrate-coating system}

Consider now a vibrating substrate (a rectangular parallelepiped with edges aligned to cartesian coordinates $\mathbf{x}_{1}, \mathbf{x}_{2}$ and $\mathbf{x}_{3}$ ) on which a thin coating is deposited (on a face normal to the $\mathbf{x}_{3}$ axis). The kinetic and potential energy of the substrate are

$$
E_{k}=\frac{1}{2} \int_{V_{s}} \rho_{s} \dot{u}_{i} \dot{u}_{i} \mathrm{~d} V_{s} \quad \text { and } \quad E_{p}=\frac{1}{2} \int_{V_{s}} C_{i j k l}^{(s)} \frac{\partial u_{i}}{\partial x_{j}} \frac{\partial u_{k}}{\partial x_{l}} \mathrm{~d} V_{s}
$$

where, $\mathbf{u}$ is the displacement field, $\rho_{s}$ is the density, $C_{i j k l}^{(s)}$ are the elastic coefficients and $V_{s}=\left\langle-d_{1} / 2 ; d_{1} / 2\right\rangle \times\left\langle-d_{2} / 2 ; d_{2} / 2\right\rangle \times\left\langle-d_{3} / 2 ; d_{3} / 2\right\rangle$ is the volume of the substrate. By taking polynomial approximation of the displacement field

$$
u_{i}=\sum_{k=1}^{N} \alpha_{k}^{(i)} \psi_{k}\left(x_{1}, x_{2}, x_{3}\right) \cos (\omega t)
$$

(where $\omega$ is the angular frequency of harmonic vibrations and the basis $\psi_{k}$ can be advantageously chosen as orthonormal Legendre polynomials), it can be easily shown (e.g. [5]) that the problem of finding stationary points of the Lagrangian of the substrate $L=E_{k}-E_{p}$ can be rewritten as a generalized eigenvalue problem of the following form:

$$
\mathbb{K} \vec{\alpha}-\omega^{2} \mathbb{M} \vec{\alpha}=\overrightarrow{0},
$$

where $\vec{\alpha}$ is a vector of coefficients from approximation (2) and the matrices $\mathbb{K}$ and $\mathbb{M}$ are given by the geometry, density and elastic coefficients of the substrate $[5,7,10,12]$. 
Let us now incorporate the presence of the coating in this model. The kinetic and potential energy of the coating (understood here as small perturbations $\delta E_{k}$ and $\delta E_{p}$ ) are considered now as fully given by the displacement field $\mathbf{u}$ at the surface of the substrate where the coating is deposited. For $h$ standing for the thickness of the coating, a possible assumption is to take this displacement field (denoted here as $\left.\mathbf{v}\left(x_{1}, x_{2}, t\right)\right)$ as an extrapolation of displacement field $\mathbf{u}$ in the midplane of the coating (e.g. in distance $h / 2$ above the surface of the substrate) (in this simplification, we neglect the fact that the derivatives of $\mathbf{u}$ can be discontinuous over the substrate-coating interface) which reads

$$
v_{i}\left(x_{1}, x_{2}, t\right)=u_{i}\left(x_{1}, x_{2}, x_{3}=d_{3} / 2, t\right)+\frac{h}{2} \frac{\partial u_{i}}{\partial x_{3}}\left(x_{1}, x_{2}, x_{3}=d_{3} / 2, t\right)
$$

for $i=1,2,3$. This leads to the following simplified expressions

$$
\delta E_{k}=\frac{h}{2} \int_{S_{c}} \rho_{c} \dot{v}_{i} \dot{v}_{i} \mathrm{~d} S_{c} \quad \text { and } \quad \delta E_{p}=\frac{h}{2} \int_{S_{c}} C_{i j k l}^{(c)} \frac{\partial v_{i}}{\partial x_{j}} \frac{\partial v_{k}}{\partial x_{l}} \mathrm{~d} S_{c}
$$

for the kinetic and elastic energy of the coating respectively, where the subscripts $c$ and superscripts $(c)$ denote the quantities related to the coating. Using the approximation (2) again, a perturbed eigenvalue problem can be arrived, relating the perturbation of matrices $\mathbb{K}$ and $\mathbb{M}$ to the small changes in the angular frequencies $\delta \omega^{2}$ as

$$
(\mathbb{K}+\delta \mathbb{K})(\vec{\alpha}+\delta \vec{\alpha})-\left(\omega^{2}+\delta \omega^{2}\right)(\mathbb{M}+\delta \mathbb{M})(\vec{\alpha}+\delta \vec{\alpha})=\overrightarrow{0} .
$$

In this first, linear approximation, is $\vec{\alpha} \perp \mathbb{M} \delta \vec{\alpha}$ and thus, after some additional algebra, the perturbation of the resonant frequency of the $i$-th mode $\left(\delta \omega_{i}^{2}\right)$ can be expressed as

$$
\delta \omega_{i}^{2}=\frac{{\overrightarrow{\alpha_{i}}}^{\mathrm{T}}\left(\delta \mathbb{K}-\omega_{i}^{2} \delta \mathbb{M}\right) \overrightarrow{\alpha_{i}}}{{\overrightarrow{\alpha_{i}}}^{\mathrm{T}} \mathbb{M} \overrightarrow{\alpha_{i}}}
$$

This formula directly relates the shifts of the resonant frequencies to the elastic coefficients, the density and the thickness of the coating (embodied here by the perturbations $\delta \mathbb{K}$ and $\delta \mathbb{M}$.) In other words, it enables $C_{i j k l}^{(c)}$ to be determined directly from the shifts $\delta \omega_{i}^{2}$, without solving the full eigenvalue problem (6) within every step of the inverse procedure. This makes the use of the perturbation model not only enormously less computation-time-consuming than the classical approaches, but more lucid for further theoretical analysis (e.g. estimation of the experimental errors).

It is more than obvious that the shifts $\delta \omega_{i}^{2}$ cannot contain sufficient information on all the elastic coefficients $C_{i j k l}^{(c)}$. In the potential energy $\delta E_{p}$, all the products $C_{i j k l}^{(c)} \varepsilon_{i j} \varepsilon_{k l}=\sigma_{k l}^{(c)} \varepsilon_{k l}$ give zero whenever $k$ or $l$ equals to 3 (plane stress conditions in the coating), which decreases the number of the involved elastic coefficients from 21 down to 6 . These coefficients can be arranged into a symmetric $3 \times 3$ matrix $\mathbb{Q}$, which represents a linear relation between the non-zero components of the stress tensor ( $\sigma_{11}, \sigma_{22}$ and $\sigma_{12}$ only) to the in-plane components of the strain 
tensor $\left(\varepsilon_{11}, \varepsilon_{22}\right.$ and $\left.\varepsilon_{12}\right)$, i.e. $\left[\sigma_{11}, \sigma_{22}, \sigma_{12}\right]^{\mathrm{T}}=\mathbb{Q}\left[\varepsilon_{11}, \varepsilon_{22}, 2 \varepsilon_{12}\right]^{\mathrm{T}}$. It can be easily shown that

$$
\mathbb{Q}=\left(\begin{array}{ccc}
\left(C^{-1}\right)_{1111}^{(c)} & \left(C^{-1}\right)_{1122}^{(c)} & \left(C^{-1}\right)_{1112}^{(c)} \\
& \left(C^{-1}\right)_{2222}^{(c)} & \left(C^{-1}\right)_{2212}^{(c)} \\
\text { symm. } & & \left(C^{-1}\right)_{1212}^{(c)}
\end{array}\right)^{-1},
$$

where $\left(C^{-1}\right)_{i j k l}^{(c)}$ is a compliance tensor. Further in this text, we will use this matrix $\mathbb{Q}$ for characterization of in-plane elastic coefficients of the coatings, i.e. of those elastic coefficients of the coatings theoretically obtainable from RUS measurements on the substrate-coating system.

\subsection{Numerical comparison to a full 3D-3D model}

The above proposed perturbation model can be expected to work properly for $h \ll$ $d_{3}$. Indeed, both the perturbations of the energetic quantities, $\delta E_{k}$ and $\delta E_{p}$, are linearly proportional to the thickness of the coating, so limiting $h \rightarrow 0$ increases the validity of our assumptions. A natural question arises, how thin the coating must be to justify the use of the perturbation model, i.e. to ensure that this model provides the frequency shifts with some satisfying accuracy. To investigate this, and in order to verify the correctness of our model, we made the following preliminary numerical tests prior to applying the perturbation model to real experimental data:

1. We considered a homogeneous specimen (a $4 \mathrm{~mm} \times 5 \mathrm{~mm} \times 1 \mathrm{~mm}$ single crystal of silicon cut along the principal directions) with the smallest dimension slightly increasing, and analyzed whether the changes of the resonant frequencies due to the thickening (evaluated by solving the eigenvalue problem (3) for different $d_{3}$ ) can be captured by the perturbation model. In other words, we analyzed a substrate-coating system, where the coating had exactly the same elastic properties as the substrate.

2. We assumed a full 3D-3D 'sandwich' model (similar to [13] but using an orthogonal basis) as a reference and compared the frequency shifts predicted by this model to those evaluated by relation (7). The analyzed system in this case was a CVD deposited diamond coating on the same substrate as in the first example.

For both these systems, shifts of the first thirty resonant frequencies of the substrate were evaluated. The results are shown in Fig. 1. In the upper row, the frequency shifts are shown for two chosen modes (the 1st and the 12th mode in the spectrum; circles denote the full 3D-3D models, solid lines are the shifts determined by relation (7)), in the lower row, the difference between the shifts predicted by the both models for all analyzed thirty modes is plotted versus the thickness and the mode number. The results are shown for the thickness of the coating ranging from $10 \mathrm{~nm}$ to $100 \mu \mathrm{m}$.

Obviously, there is a significant difference between the two analyzed systems. Except of three or four modes, the perturbation model sufficiently approximates 
(a) Si coating on a Si substrate
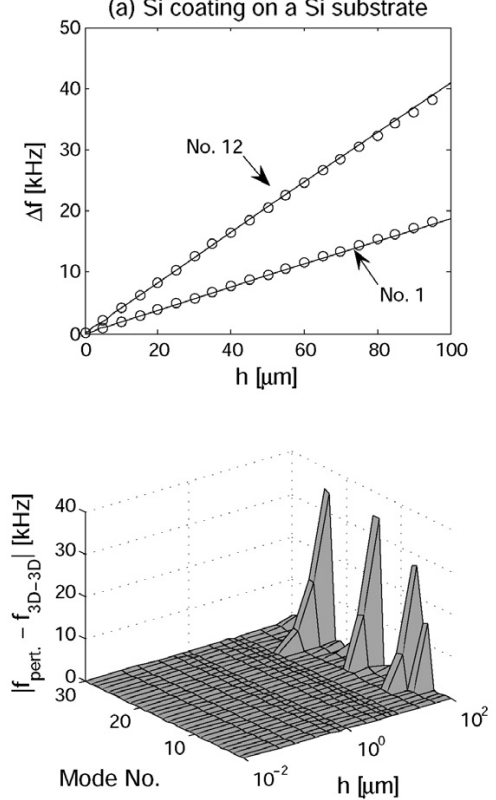
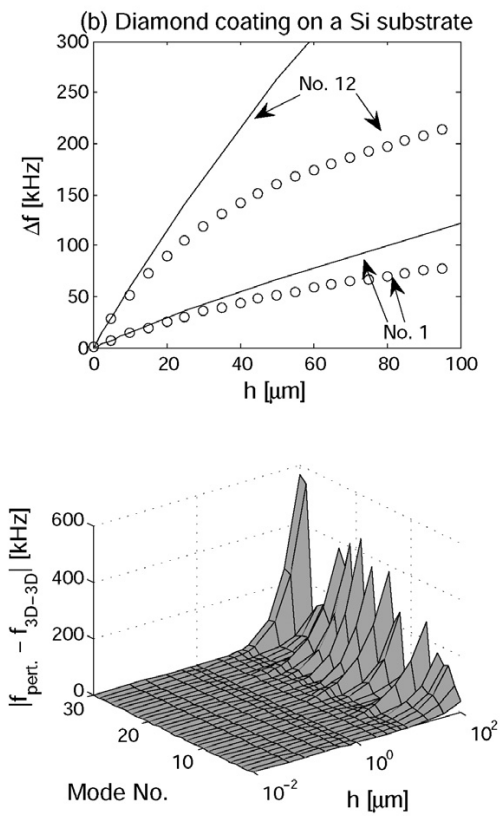

Figure 1: Comparison of the perturbation method to a full 3D-3D model. Upper row: Frequency shifts for the $1^{\text {st }}$ and the $12^{\text {th }}$ mode depending on the thickness of the coating $h$. (Circles denote the full 3D-3D models, solid lines the perturbation method). Lower row: Differences between the two discussed models for the first 30 modes.

the frequency shifts for the silicon substrate with changing thickness up to $h=$ $100 \mu \mathrm{m}$, whereas the silicon-diamond system is reasonably described by the perturbation model for $h \leq 10 \mu \mathrm{m}$ only. The reason may be found in the disproportion between the elastic properties of silicon and of the deposited diamond. The cubic single crystal of silicon has $c_{11}=166 \mathrm{GPa}, c_{12}=64 \mathrm{GPa}$ and $c_{44}=80 \mathrm{GPa}$; for the isotropic diamond coating (polycrystal aggregate), the bulk coefficients $c_{11}=1143 \mathrm{GPa}$ and $c_{44}=530 \mathrm{GPa}$ were taken from [7]. Thus the diamond is nearly seven times 'tougher' in tension than the silicon substrate itself. That is the reason why the potential energy of a $100 \mu \mathrm{m}$ thick diamond coating cannot be ever taken as a small perturbation of the potential energy of the $1 \mathrm{~mm}$ thick silicon substrate, and the perturbation approach fails. We can conclude that any estimation of limiting thickness $h$ for which the perturbation approach reliably works is impossible without taking the ratio between elastic moduli of the substrate and the coating into account. 


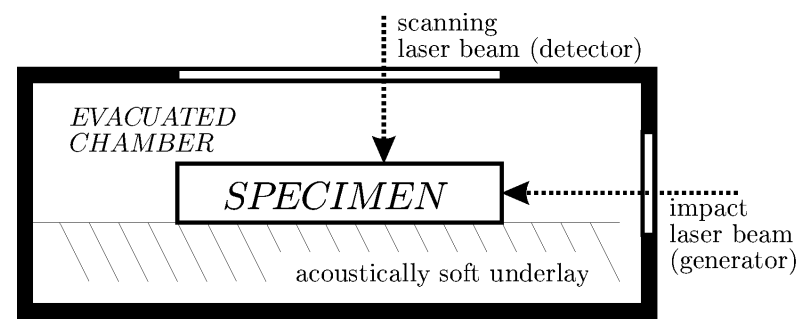

Figure 2: Outline of the non-contact experimental scheme of RUS.

\section{Experiment}

In this final section, the above outlined approach will be verified experimentally. The experiment (the setup and instrumentation) will be briefly described, and the results for two testing materials will be presented and discussed.

\subsection{Experimental setup}

For the RUS measurements described in this paper, a fully non-contact scheme [11] was used. In this scheme, the specimen is excited by an impact of a focused laser pulse (so-called thermoacoustic source) and the vibrations are detected by laserDoppler interferometer scanning the surface of the excited specimen. The specimen itself is laid of an underlay which must be extremely acoustically soft (i.e. its acoustic impedance must be incomparably smaller than the impedance of the specimen) to ensure a good approximation of the free-surface boundary conditions. To minimize the damping of the specimen, the whole measurement is performed in an evacuated chamber with two silica-glass windows, one for each of the used laser beams (see Fig. 2 for an outline). The used instrumentation was following: The elastic vibrations in the specimen were excited by sequences of pulses of a focused infrared laser beam (pulse duration $8 \mathrm{~ns}$, energy $25 \mathrm{~mJ}$, Quantel ULTRA Nd:YAG Laser system, equipped by fiber optic - FOLA options). The displacement response was detected in a mesh of points on the sample surface by Polytec Micro System Analyzer MSA-500 (using the OFV-5000 controller and the sensor head OFV-551).

This scheme was inspired by measurements by Zadler [19], who used lasers to excite specimens hanging on thin silica wires. However, the use of the acoustically soft underlay proposed by the authors seems to provide adequately good approximation of the free-surface conditions, but with much easier specimen mounting and replacement. This experimental concept was already successfully applied to determine elastic coefficients of various bulk materials [11,12], and was shown to be especially suitable for quantitative measurements of ultrasound attenuation [20]. 


\subsection{Experimental verification of the perturbation model}

The non-contact experimental scheme of RUS was used to verify the perturbation model. To know the sought elastic coefficients of the examined layers with the highest possible accuracy, and thus, to be able to analyze how reliably these coefficients can be determined by relation (7), we decided to use the same approach as in the first of the preliminary numerical tests, i.e. to perform the measurements on homogeneous specimens where the presence (or absence) of the surface coating is represented by a change in thickness.

Two different materials were used for such experiments. The first was a single crystal of silicon $(a) .1 \mathrm{~mm} \times 3.6 \mathrm{~mm} \times 3.1 \mathrm{~mm}$ rectangular parallelepiped cut along the principal crystallographic axes) and the second a reaction-bonded silicon carbide $\mathrm{Si}-\mathrm{SiC}(1.8 \mathrm{~mm} \times 2.5 \mathrm{~mm} \times 2.8 \mathrm{~mm}$ rectangular parallelepiped $)$. These specimens were taken as substrates with surface layers; the resonant spectra of these specimens were measured (covering always about the first thirty modes) and individual modes of vibration within the spectra were identified. After that, the considered surface layers were removed by polishing: $32 \mu \mathrm{m}$ thick layer was polished out from the single crystal of silicon, $8 \mu \mathrm{m}$ thick layer from the specimen of the reaction-bonded silicon carbide. This approach simulated the issue of determination of elastic properties of a $32 \mu \mathrm{m}$ thick cubic surface coating and a $8 \mu \mathrm{m}$ thick isotropic surface coating. The resonant spectra of the specimens without the 'coatings' were measured and the frequency shifts $\delta \omega^{2}$ for particular modes were identified (in this point, the identification of the modes from scanning laserDoppler interferometry is obviously crucial, as the original order of the resonances can be shuffled when the layer is deposited/removed).

To verify the reproducibility of the measurements, the spectra of the specimens before the layers were polished out were measured repeatedly under slightly different conditions (the impacting laser was was focused on different places of the specimen, the specimen was removed from the chamber and put back again etc.). In Fig. 3, the spectra obtained by two different measurements of the specimen before polishing (solid lines) are compared to the spectrum of the specimen without the examined layer. Obviously, the reproducibility of the measurements is extremely good and all the shifts of individual peaks in the spectrum can be reliably ascribed to the presence/absence of the surface layer.

Our aim was to determine the elastic coefficients of the removed layers $Q_{i j}$ by inverting the perturbation formula (7). This was done in two different ways:

1. The symmetry class of the examined material was considered as known, i.e. only three independent coefficients for cubic silicon were sought $\left(Q_{11}, Q_{12}\right.$ and $\left.Q_{33}\right)$ and two $\left(Q_{11}\right.$ and $\left.Q_{33}\right)$ for the isotropic silicon carbide.

2. Full elastic anisotropy of the layer was considered, which means six independent elastic coefficients $Q_{i j}$. However, as all the modes of vibration of the examined layer were forced by free vibrations of the specimen, the layers were loaded only in modes having the same symmetry as the substrates (i.e. in modes symmetric or antisymmetric with respect to symmetry planes of the substrates). This obviously precludes reliable determination of the coef- 


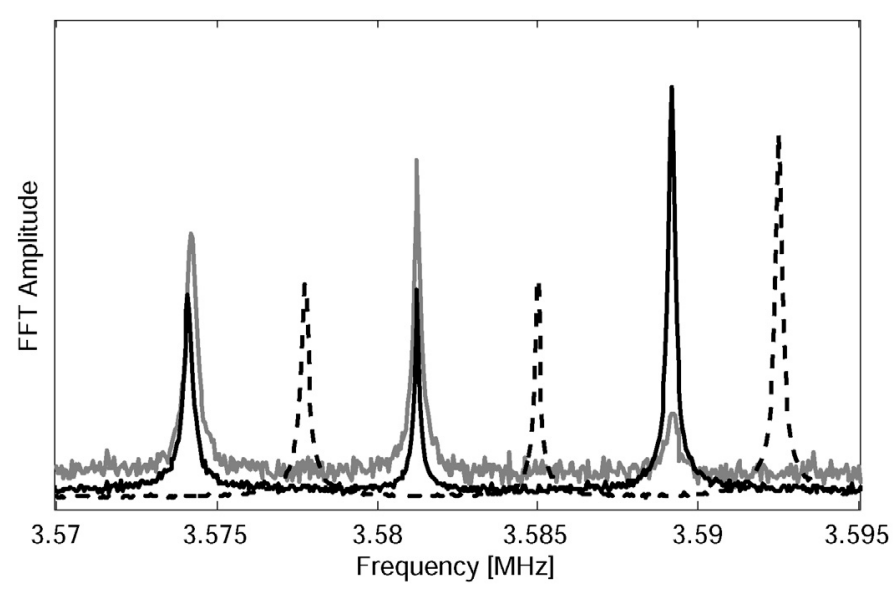

Figure 3: A selected part of the obtained spectrum ( $\mathrm{Si}-\mathrm{SiC}$ specimen) illustrating the reproducibility of the non-contact RUS measurements and the shifts induced by the absence of the surface layer. Solid lines (black and gray) correspond to two independent measurements of the original specimen, the dashed line shows how the resonant frequencies change after a $8 \mu \mathrm{m}$ thick surface layer was removed.

ficients $Q_{13}$ and $Q_{23}$, so only $Q_{11}, Q_{22}, Q_{12}$ and $Q_{33}$ were sought. (The coefficients $Q_{13}$ and $Q_{23}$ could be, however, determined by measurements on less symmetric substrates.)

In both cases, the sought coefficients $Q_{i j}$ were calculated by numerical inversion of relation (7), whereto the measured shifts of resonant frequencies were fitted in the least-square sense. In Tab. 1, the results are listed and compared to coefficients $Q_{i j}^{(s)}$ determined from the elastic coefficients of the substrate $C_{i j k l}^{(s)}$ by formula (8). The experimental errors displayed in Tab. 1 result from the accuracy of measurements of the removed layer, which was estimated to be about $1 \mu \mathrm{m}$.

In the case of the single crystal of silicon, this table shows how powerful tool the perturbation model can be. Not only that all the elastic coefficient for the known class of symmetry were determined with satisfying accuracy (especially when the uncertainty given by the thickness of the layer is taken into account), but also the cubic symmetry can be reliably identified: For cubic silicon, the constants $Q_{11}$ and $Q_{22}$ differ by less than $2 \%$. For silicon carbide, the results are less satisfying. Although for the given symmetry class, the results are quite good, the isotropy requires $Q_{11}=Q_{22}=Q_{12}+2 Q_{33}$, which is here not fulfilled (difference larger than $10 \%$ ). This may be ascribed to heterogeneity or porosity of the material or to the fact that for $h=8 \mu \mathrm{m}$, the uncertainty in the thickness (and plan-parallelism) of the removed layer is more significant. 
246 Computational Methods and Experiments in Materials Characterisation IV

\begin{tabular}{|c|c|c|c|c|c|c|c|}
\cline { 3 - 8 } \multicolumn{2}{c|}{} & \multicolumn{3}{c|}{ Bulk material } & \multicolumn{3}{c|}{ Layer (perturbation model) } \\
\hline $\begin{array}{c}\text { Examined } \\
\text { material }\end{array}$ & $\begin{array}{c}h \\
{[\mu \mathrm{m}]}\end{array}$ & $\begin{array}{c}Q_{11}^{(s)} \\
{[\mathrm{GPa}]}\end{array}$ & $\begin{array}{c}Q_{12}^{(s)} \\
{[\mathrm{GPa}]}\end{array}$ & $\begin{array}{c}Q_{33}^{(s)} \\
{[\mathrm{GPa}]}\end{array}$ & $\begin{array}{c}Q_{11} \\
{[\mathrm{GPa}]}\end{array}$ & $\begin{array}{c}Q_{12} \\
{[\mathrm{GPa}]}\end{array}$ & $\begin{array}{c}Q_{33} \\
{[\mathrm{GPa}]}\end{array}$ \\
\hline Silicon & $\begin{array}{c}32 \\
\pm 1\end{array}$ & 141.5 & 39.5 & 79.7 & $\begin{array}{c}142.0 \\
\pm 4.3\end{array}$ & $\begin{array}{c}37.4 \\
\pm 1.2\end{array}$ & $\begin{array}{c}82.8 \\
\pm 2.6\end{array}$ \\
\hline Si-SiC & $\begin{array}{c}8 \\
\pm 1\end{array}$ & 412.2 & -- & 168.6 & $\begin{array}{c}436.2 \\
\pm 54.5\end{array}$ & -- & $\begin{array}{l}192.7 \\
\pm 24.1\end{array}$ \\
\hline
\end{tabular}

\begin{tabular}{|c|c|c|c|c|c|c|c|}
\cline { 3 - 8 } \multicolumn{2}{c|}{} & \multicolumn{6}{|c|}{ Layer (perturbation model, full anisotropy) } \\
\hline $\begin{array}{c}\text { Examined } \\
\text { material }\end{array}$ & $\begin{array}{c}h \\
{[\mu \mathrm{m}]}\end{array}$ & $\begin{array}{c}Q_{11} \\
{[\mathrm{GPa}]}\end{array}$ & $\begin{array}{c}Q_{12} \\
{[\mathrm{GPa}]}\end{array}$ & $\begin{array}{c}Q_{13} \\
{[\mathrm{GPa}]}\end{array}$ & $\begin{array}{c}Q_{22} \\
{[\mathrm{GPa}]}\end{array}$ & $\begin{array}{c}Q_{23} \\
{[\mathrm{GPa}]}\end{array}$ & $\begin{array}{c}Q_{33} \\
{[\mathrm{GPa}]}\end{array}$ \\
\hline Silicon & 32 & 140.9 & 36.4 & N/A & 143.1 & N/A & $\begin{array}{l}82.8 \\
\pm 2.2\end{array}$ \\
& \pm 1 & \pm 4.4 & \pm 1.1 & & \pm 4.5 & & \pm 2.2 \\
\hline $\mathrm{Si}-\mathrm{SiC}$ & 8 & 422.4 & 32.6 & N/A & 454.4 & N/A & 179.0 \\
& \pm 1 & \pm 52.8 & \pm 4.1 & & \pm 56.8 & & \pm 22.4 \\
\hline
\end{tabular}

Table 1: Comparison of in-plane elastic coefficients $Q_{i j}$ determined by RUS on bulk specimens and by the perturbation model.

\section{Conclusion}

By introducing the perturbation theory into the RUS of thin surface coatings, the in-plane elastic coefficients of isotropic and cubic coatings can be easily and reliably determined. In future, the authors would like to focus on the effect of the disproportion between the elastic properties of the substrate and the coating (see paragraph 2.2) and the the analysis of the experimental errors of the obtained coefficients in dependence on various factors, such as symmetry of the substrate, etc.

\section{Acknowledgements}

This work was financially supported by the Czech Science Foundation (project No.202/09/P164), the Grant Agency of ASCR (project No. A200100627), the Czech Ministry of Education (project No. 1M06031) and the institutional project of IT ASCR CEZ: AV0Z20760514.

\section{References}

[1] Soga, N. \& Anderson, O.L., Elastic properties of tektites measured by resonant sphere technique. Journal of Geophysical Research, 72, pp. 1733-1739, 1967.

[2] Demarest, H.H., Cube resonance method to determine the elastic constants of solids. Journal of Acoustical Society of America, 49(3 pt 2), pp. 768-775, 1971.

[3] Ohno, I., Free vibration of a rectangular parallelepiped crystal and its application to determination of elastic constants of orthorhombic crystals. Journal of Physics of the Earth, 24, pp. 355-379, 1976. 
[4] Ohno, I., Yamamoto, S., Anderson, O.L. \& Noda, J., Determination of elastic constants of trigonal crystals by the rectangular parallelepiped resonance method. Journal of Physics and Chemistry of Solids, 47(12), pp. 1103-1108, 1986.

[5] Migliori, A., Sarrao, J.L., Visscher, W.M., Bell, T.M., Lei, M., Fisk, Z. \& Leisure, G.R., Resonant utrasound spectroscopy techniques for measurements of the elastic moduli of solids. Physica B, 183(1-2), pp. 1-24, 1993.

[6] Lei, M., Sarrao, J., Visscher, W., Bell, T., Thompson, J., Migliori, A., Welp, U. \& Veal, B.W.H., Elastic constants of a monocrystal of superconducting YBa2Cu3O7- $\delta$. Physical Review B, 47(10), pp. 6154-6156, 1993.

[7] H.Ogi, Sato, K., Asada, T. \& Hirao, M., Complete mode identification for resonance ultrasound spectroscopy. Journal of Acoustical Society of America, 112(6), pp. 2553-2557, 2002.

[8] Landa, M., Sedlák, P., Šittner, P., Seiner, H. \& Novák, V., Temperature dependence of elastic properties of cubic and orthorhombic phases in $\mathrm{Cu}-\mathrm{Al}-\mathrm{Ni}$ shape memory alloy near their stability limits. Materials Science and Engineering A, 482(1-2), pp. 320-324, 2007.

[9] Landa, M., Sedlák, P., Šittner, P., Seiner, H. \& Heller, L., On the evaluation of temperature dependence of elastic constants of martensitic phases in shape memory alloys from resonant ultrasound spectroscopy studies. Materials Science and Engineering A, 481-482(1-2 C), pp. 567-573, 2008.

[10] Landa, M., Sedlák, P., Seiner, H., Heller, L., Bicanová, L., Šittner, P. \& Novák, V., Modal resonant ultrasound spectroscopy for ferroelastics. Applied Physics A: Materials Science and Processing, pp. 1-11, Article in Press, 2009.

[11] Sedlák, P., Landa, M., Seiner, H., Bicanová, L. \& Heller, L., Non-contact resonant ultrasound spectroscopy for elastic constants measurement. 1st International Symposium on Laser Ultrasonics: Science, Technology and Applications. Available online at www.ndt.net. pp. 1-6, 2008.

[12] Landa, M., Seiner, H., Sedlák, P., Bicanová, L., Zídek, J. \& Heller, L., Resonant ultrasound spectroscopy close to its applicability limits. Horizons in World Physics, Volume 246, eds. M. Everett \& L. Pedroza, Nova Publishers: New York, Expected publication date: 2nd quarter of 2009.

[13] Visscher, W.M., Migliori, A., Bell, T.M. \& Reinert, R.A., On the normal modes of free vibration of inhomogeneous and anisotropic elasti objects. Journal of Acoustical Society of America, 90(4), pp. 2154-2162, 1991.

[14] So, J.H., Gladden, J.R., Hu, Y.F., Maynard, J.D. \& Li, Q., Measurements of elastic constants in thin films of colossal magnetoresistance material. Physical Review Letters, 90(3), pp. 036103-1-036103-4, 2003.

[15] Nakamura, N., Ogi, H. \& Hirao, M., Determination of anisotropic elastic constants of superlattice thin films by resonant ultrasound spectroscopy. Journal of Applied physics, 97(1), pp. 013532-1-013532-6, 2005.

[16] Nakamura, N., Ogi, H. \& Hirao, M., Elastic constants of chemical-vapordeposition diamond thin films: resonance ultrasound spectroscopy with laserdoppler interferometry. Acta Materialia, 52(3), pp. 765-771, 2004. 
248 Computational Methods and Experiments in Materials Characterisation IV

[17] Nakamura, N., Ogi, H. \& Hirao, M., Resonance ultrasound spectroscopy with laser-doppler interferometry for studying elastic properties of thin films. Ultrasonics, 42(1-9), pp. 491-494, 2004.

[18] P.Heyliger, Traction-free vibration of layered elastic and piezoelectric rectangular parallelepipeds. Journal of Acoustical Society of America, 107(3), pp. 1253-1245, 2000.

[19] Zadler, B.J., PhD Thesis. Colorado School of Mines, 2005.

[20] Seiner, H., Bicanová, L., Sedlák, P., Landa, M., Heller, L. \& Aaltio, I., Magneto-elastic attenuation in austenitic phase of Ni-Mn-Ga alloy investigated by ultrasonic methods. Accepted to Materials Science and Engineering $A, 2008$. Article in Press. 